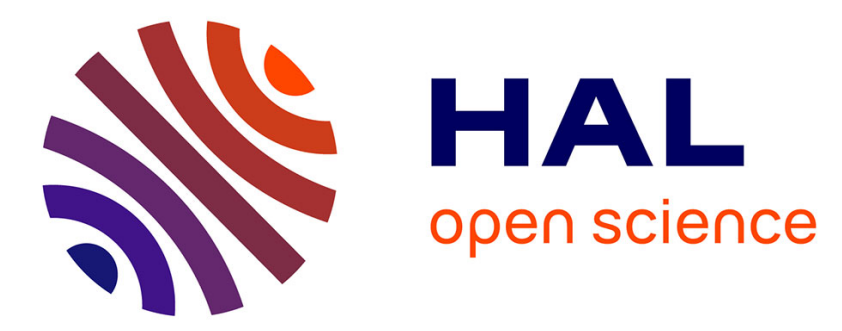

\title{
FORCES DUE TO DIFFRACTION OF SOUND WAVE ON SMALL-DIAMETER CYLINDRICAL PARTICLES
}

\author{
H. Czyz, T. Gudra
}

\section{To cite this version:}

H. Czyz, T. Gudra. FORCES DUE TO DIFFRACTION OF SOUND WAVE ON SMALLDIAMETER CYLINDRICAL PARTICLES. Journal de Physique IV Proceedings, 1992, 02 (C1), pp.C1-741-C1-744. 10.1051/jp4:19921161 . jpa-00251121

HAL Id: jpa-00251121

https://hal.science/jpa-00251121

Submitted on 1 Jan 1992

HAL is a multi-disciplinary open access archive for the deposit and dissemination of scientific research documents, whether they are published or not. The documents may come from teaching and research institutions in France or abroad, or from public or private research centers.
L'archive ouverte pluridisciplinaire HAL, est destinée au dépôt et à la diffusion de documents scientifiques de niveau recherche, publiés ou non, émanant des établissements d'enseignement et de recherche français ou étrangers, des laboratoires publics ou privés. 


\title{
FORCES DUE TO DIFFRACTION OF SOUND WAVE ON SMALL-DIAMETER CYLINDRICAL PARTICLES
}

\author{
H. CZYZ and T. GUDRA*
}

Institute of Physics, Pedagogical University of Rzeszów, uL Rejtana 16A, PL-35-310 Rzeszow, Poland ${ }^{*}$ Ecole Polytechnique, PL-50-317 Wroclaw, Poland

\begin{abstract}
In the paper we investigate the problem of diffraction of plane sound wave on small-diameter rigid cylindrical particles of arbitrary orientation. We estimate amplitudes of force and torque due to the diffraction phenomena. Some predictions are made concerning the acoustic radiation force on such particles.
\end{abstract}

Sommaire. - Nous avons analysé la théorie de l'action d'un champ acoustique stationnaire sur des particules en forme de cylindre. On observe deux effects de la pression de radiation: une réorientation des cylindres parallelement aux plans d'onde et un regroupement des cylindres dans les plans nodaux de pression acoustique. Nous avons essayé de trouver une expression: $1^{\circ}$ de la force par tels cylindres en fonction de l'angle; $2^{\circ}$ de la couple en fonction de l'angle.

\section{Introduction}

Although many papers were published on the acoustic radiation pressure on disks and spheres, which are commonly used as test targets in calibration of ultrasonic fields, the analogous problems for cylinders met very limited interest. Some progress has ben done recently in connection with two technical applications: acousto-optical examination of dilute water suspensions of paper-making fibres (see e. g. [1] and References therein) and acoustic control of forces used in some precision biophysical experiments [2].

A theoretical approach has also been done concerning the acoustic radiation force on elastic cylinders [3]. In most analyses, however, only the case of a cylinder perpendicular to the wave vector is considered. In this paper we consider the problem of diffraction of plane progressive wave on a fixed, rigid, small-diameter cylinder of arbitrary orientation. In conclusion, we make some remarks on the angular dependence of the acoustic radiation force experienced by a freely suspended cylinder in a standing sound wave.

\section{The diffraction problem}

We consider an infinitely long cylider of radius $a$; its axis coincides with the $z$ axis of an orthogonal coordinate system $(x, y, z)$. The incident plane wave is represented by the wave vector $\vec{k}$, while $\omega=k c$ is the angular frequency. The acoustic potential of the wave can be written as

$$
\Phi_{I}=\Phi_{0} \exp (i \omega t-i \vec{k} \vec{R}) .
$$




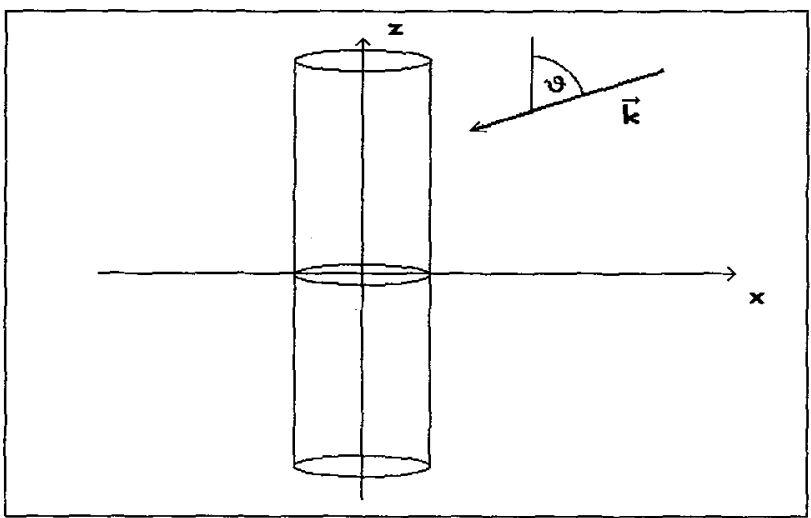

Fig. 1

We assume the geometry as presented in Fig. 1 . Therefore we put $\vec{k} \vec{R}=-k_{x} x-k_{z} z$, where $k_{x}=k \sin \vartheta$ and $k_{z}=k \cos \vartheta$. In the cylindrical system $(r, \varphi, z)$ such that $x=r \cos \varphi, y=r \sin \varphi$, we can rewrite Eq. (1) as

$$
\Phi_{I}=\Phi_{0} \exp (i \omega t) \exp (i k z \cos \vartheta) \exp (i k r \sin \vartheta \cos \varphi) .
$$

To separate cylindrical coordinates $r$ and $\varphi$, we represent the last exponential function as the well-known series of the Bessel functions:

$$
\Phi_{I}=\Phi_{0} \exp (i \omega t) \exp (i k z \cos \vartheta) \sum_{m=0}^{\infty} c_{m} \cos (m \varphi) J_{m}(k r \sin \vartheta)
$$

where $c_{0}=1, c_{m}=2 i^{m}$ for $m>0$. It is natural to look for the reflected wave in the form

$$
\Phi_{R}=\Phi_{0} \exp (i \omega t) \exp (i k z \cos \vartheta) \sum_{m=0}^{\infty} d_{m} \cos (m \varphi) H_{m}^{(2)}(k r \sin \vartheta)
$$

To determine coefficients $d_{m}$, we apply the boundary condition for a rigid, fixed cylinder:

$$
\left.\frac{\partial\left(\Phi_{I}+\Phi_{R}\right)}{\partial r}\right|_{r=a}=0
$$

It is easy to demonstrate, that the total acoustic potential $\Phi_{I}+\Phi_{R}$ is

$$
\Phi=\Phi_{0} \exp (i \omega t) \exp (i k z \cos \vartheta) \sum_{m=0}^{\infty} d_{m} \cos (m \varphi)\left[J_{m}(k r \sin \vartheta)-\frac{J_{m}^{\prime}(k a \sin \vartheta)}{H_{m}^{(2)^{\prime}}(k a \sin \vartheta)}\right] H_{m}^{(2)}(k r \sin \vartheta)
$$

Using basic properties of Bessel functions we obtain at the surface of the cylinder

$$
p=p_{0} \exp (i \omega t) \exp (i k z \cos \vartheta) \frac{2}{i \pi k a \sin \vartheta} \sum_{m=0}^{\infty} c_{m} \frac{\cos (m \varphi)}{H_{m}^{(2)^{\prime}}(k a \sin \vartheta)}
$$

(we made use of the relation $p=i \omega \Phi)$. 
The above formula may be used to calculate the amplitude of the force on the cylinder. As we are interested mainly in the case of small-diameter particles, we can limit ourselves to terms of order of $(k a)^{1}$. After some calculations we get $[5]$

$$
\left.p=p_{0} \exp (i \omega t) \exp (i k z \cos \vartheta)[1+i 2 k a \sin \vartheta \sin \varphi)\right] .
$$

The amplitude of the force is the integral of the pressure over the surface of the cylinder. The symmetry of the problem indicates that the force has only the $x$-component. As $d s_{x}=a \cos \varphi d \varphi d z$, the force on a cylinder of length $2 l$ is

$$
F=-\int_{-l}^{l} \int_{0}^{2 \pi} p a \cos \varphi d \varphi d z
$$

Using Eq. (8) we get for small $k a$

$$
F=-i \exp (i \omega t) S p_{0} k a \sin \vartheta \frac{\sin (k l \cos \vartheta)}{k l \cos \vartheta}
$$

where $S=4 \pi a l$ is the side area of the cylinder.

It is also the symmetry that indicates that the torque on the cylinder has only the $y$-component. Integrated the quantity $p z d s_{x}$ over the cylinder surface one obtains for the torque

$$
M_{y}=\exp (i \omega t) S p_{0} l \frac{k a \sin \vartheta}{k l \cos \vartheta}\left[\frac{\sin (k l \cos \vartheta)}{k l \cos \vartheta}-\cos (k l \cos \vartheta)\right] .
$$

It is easy to see that in the case $\vartheta=\pi / 2$, i. e. when the wave vector is perpendicular to the particle axis, the torque vanishes, while the amplitude of the force becomes $S p_{0} k a$.

\section{Acoustic radiation pressure}

The problem of the drift force related to second order phenomena in acoustic field has been studied by Hasegawa et. al. [3] and by Wu et. al. [2]. In both papers oly the case of perpendicular incidence has been considered. Here we limit ourselves to some remarks on the dependence of the radiation pressure force on the angle of incidence of the wave.

According to King [5], the excess pressure in the acoustic field up to second order can be written as

$$
p=\rho_{0} d \Phi / d t+\frac{\rho_{0}}{2 c^{2}}[d \Phi / d t]^{2}+\frac{\rho_{0} v^{2}}{2},
$$

where $\rho_{0}$ is the static density of the medium, $v$ is the acoustic velocity of the medium and $c$ is the speed of sound. The procedure described by King is as follows: to find the acoustic radiation force, one has to average the force on the particle:

$$
\vec{F}_{\text {dritf }}=-<\int_{S} p d \vec{s}>
$$

The acoustic pressure has to be calculated from Eq. (12), supposed the acoustic potential is a solution of the diffraction problem taking into account that the particle is in motion with respect to the medium. Therefore one has to make use of the relation

$$
d \Phi / d t=D \Phi / D t+\vec{v} \cdot \vec{u},
$$

where $\vec{u}$ is the velocity of the particle, which is to be determined from the equation of motion of the particle of unit length ( $\rho_{p}$ detotes particle density):

$$
\int_{S} p d \vec{s}=-\rho_{p} \pi a^{2} d \vec{u} / d t
$$


One has also to make use of the boundary condition at the surface of the particle in motion:

$$
-\partial \Phi /\left.\partial r\right|_{S}=\vec{u} \cdot \vec{s}
$$

To solve the problem up to second order, it is sufficient to take in the above equation only the leading term in the pressure in Eq. (12). The equation of motion becomes after integration with respect to $t$

$$
\frac{\rho_{0} / \rho_{p}}{\pi a} \int_{S} \Phi d \vec{s}=-\vec{u}
$$

The problem formulated above has been solved by $\mathrm{Wu}$ et. al. [2] for the case of perpenicularly incident wave of arbitrary form, (only the symmetry with respect to the $x$-axis was assumed). In our case of interest the wave vector has also the $z$-component, but as we demonstrated in Section 2 , the force on small-diameter cylinder still has only the $x$-component. Therefore we can put $u_{x}=u$ and consider the problem as one-dimensional.

In [2] the following expressions for the radiation pressure drift force in a standing wave has been obtained:

$$
F_{\text {drift }}=\left[\frac{1-\rho_{0} / \rho_{p}}{2\left(1+\rho_{0} / \rho_{p}\right)}+1\right] V k \frac{p_{0}^{2}}{\rho_{0} c^{2}} \sin (2 k x),
$$

where $V$ is volume of the cylinder of unit length. The factor $\sin (2 k x)$ is the same as expressions for drift forces of other origin [6]. For a particle of length $2 l$, as considered in Section 2 , and by use of our symbols we may rewrite Eq. (18) in the form

$$
F_{\text {drift }}=\left[\frac{1-\rho_{0} / \rho_{p}}{2\left(1+\rho_{0} / \rho_{p}\right)}+1\right] \frac{p_{0}^{2}}{2 \rho_{0} c^{2}} S k a \sin (2 k x)
$$

One can compare this second-order result with Eq. (10) expressing the amplitude of force on fixed cylinder. It is evident that the factor $\sin (k l \cos \vartheta) / k l \cos \vartheta$ comes from integration over cylinder's surface with respect to $z$, while the factor $k a \sin \vartheta$ stands for $k a$ in the case of perpendicular incidence. Therefore we suggest that in the case presented in Fig. 1 the radiation pressure force can be expressed as

$$
F_{\text {drift }}=\left[\frac{1-\rho_{0} / \rho_{p}}{2\left(1+\rho_{0} / \rho_{p}\right)}+1\right] \frac{p_{0}^{2}}{2 \rho_{0} c^{2}} S k a \sin \vartheta \frac{\sin (k l \cos \vartheta)}{k l \cos \vartheta} \sin (2 k x)
$$

The dependence of radiation pressure force given by Eq. (2) on $p_{0}^{2}$ has been examined experimentally by Wu et. al in [2] and a very good agreement with the theory has been observed. We plan an independent experiment to test the depenedence of the force on particle's angular position provided by Eq. (20).

\section{References}

[1] Brodeur, P., Motion of fluid-suspended fibres in a standing wa ve field. Ultrasonics 29, 302-307 (1991).

[2] Wu, J., Du, G., Work, S. S., and Warshaw, D. M., Acoustic radiation pressure on a rigid cylinder: An analytical theory and experiment. J. Acoust. Soc. Am. 87, 581-586 (1990).

[3] Hasegawa, T., Kyosuke, S., Inoue, N., and Matsuzawa K., Acoustic radiation force experienced by a solid cylinder in a plane progressive field. J. Acoust. Soc. Am. 83, 1770-1775 (1988).

[4] King, L. V., On the acoustic radiation pressure on spheres. Proc. R. Soc. Lodon Ser. A 147, 212-240 (1934).

[5] Czyż, H., Sily związane z ugięciem plaskiej fali na walcu (in Polish, English abstract). Proceedings of Open Seminar on Acoustics, Gdańsk 1990.

[6] Czyż, H., Oszacowanie wartości siły dryfu radiacyjnego dla cząstek cylindrycznych (in Polish, English abstract). Proceedings of The Open Seminar on Acoustics, Poznań 1991. 